\title{
Simultaneous integrated boost using stereotactic radiosurgery for resected brain metastases: rationale, dosimetric parameters, and preliminary clinical outcomes
}

\author{
Mark J Amsbaugh, MD, ${ }^{a}$ Neal E Dunlap, MD, ${ }^{a}$ Warren Boling, MD, ${ }^{b}$ Akanksha Rajeurs, \\ $\mathrm{BS},{ }^{\mathrm{c}}$ Timothy Y Guan, $\mathrm{PhD},{ }^{\mathrm{a}}$ Keith Sowards, $\mathrm{MS},{ }^{\mathrm{a}}$ and Shiao Woo, $\mathrm{MD}^{\mathrm{a}}$
}

Departments of ${ }^{a}$ Radiation Oncology, ${ }^{b}$ Neurosurgery, and ${ }^{\mathrm{c} S}$ chool of Medicine, University of Louisville, Louisville, Kentucky

\begin{abstract}
Background Radiosurgery has been shown to reduce the rates of local recurrence in the postoperative bed after the resection of brain metastases, but the ideal radiation dose has not been well defined.

Objective To present dosimetric parameters and preliminary clinical outcomes for patients undergoing postoperative stereotactic radiosurgery (SRS) with simultaneous integrated boost (SIB) for brain metastases.

Methods and materials 3 patients underwent surgery for a dominant metastatic focus and had residual or recurrent disease in the resection cavity. Our technique delivered a low dose to the resection cavity with an SIB dose to the gross tumor. Clinical target volume (CTV) was the magnetic resonance (MR)-defined resection cavity. Gross tumor volume (GTV) was the MR-defined residual disease. No additional margin was added to either the resection cavity or the residual disease area. Doses ranged from 14-15 Gy for CTV and 17-18 Gy for GTV prescribed to the 71\%-78\% isodose line. A traditional postoperative radiosurgery plan was constructed for each patient, and dosimetric values were compared using the paired t-test.

Results 3 patients were treated at our institution using SRS with SIB. No patient experienced local recurrence. 2 patients developed distant brain failure (mean, 3.5 months). No grade 3 or greater toxicities were observed. The volume of brain receiving 12 $G y$ was significantly reduced using SIB compared with traditional postoperative SRS $(P=.04)$. There were no differences in the maximum dose delivered to the tumor $(P=.15)$ and cavity $(P=.13)$. The average mean cavity dose was 16.20 Gy using the SIB plan, compared with $19.71 \mathrm{~Gy}$ using the traditional plan $(P=.05)$.

Conclusions In patients with either recurrent or residual disease following surgical resection, SRS using SIB is technically feasible and safe.
\end{abstract}

A $n$ estimated 9\%-26\% of cancer patients develop a brain metastasis, making it one of the most common neurologic complications of cancer. ${ }^{1,2}$ The incidence of clinically recognized brain metastases will increase as modern oncologic therapies increase survival and improved imaging detects smaller brain lesions.

Traditionally, whole brain radiation therapy (WBRT) is used to treat patients with brain metastases; however, alternative treatments are quickly evolving because of a rapid improvement in techniques, technology, and image guidance. A large percentage of patients present with a single brain metastasis; and in these cases, therapy may be local- ized, omitting treatment of the entire brain. ${ }^{3,4}$ When compared with WBRT alone, surgical resection and radiosurgery are local treatments that improve local control, overall survival, and functional outcomes in patients. ${ }^{5-7}$ In patients with limited intracranial disease, evidence suggests radiosurgery may be used alone, omitting WBRT, if these patients are closely monitored and can accept higher rates of distant brain failure. ${ }^{8-10}$

Even with high rates of local control with radiosurgery, there are many instances when surgical resection is either necessary or advantageous. Surgery can provide diagnostic information, faster symptomatic relief, better local control with larger

Accepted for publication April 29, 2015. Correspondence: Neal E Dunlap, MD; nedunl01@louisville.edu. Disclosures: The authors have no disclosures. JCSO 2015;13:214-218. C2015 Frontline Medical Communications. DOI 10.12788/ jeso.0140. 
lesions, and emergency decompression. A drawback of surgical resection as a sole modality is the high rate of local failure (range, 46\%-59\%.) ${ }^{10,11}$ In patients who undergo surgical resection of a brain metastasis, Patchell and colleagues have demonstrated that the use of WBRT decreases local failure and rates of neurologic death, and it is considered the standard of care. ${ }^{11}$ There is more interest in combining the reduced side-effect profile of stereotactic radiosurgery (SRS) with the increased local control of radiotherapy in patients who have undergone a resection for metastatic disease to the brain. ${ }^{12-31}$

The use of SRS as an adjuvant treatment is a new approach. To our knowledge, no randomized phase 3 trials have been published to date, despite the 2014 publication of the first prospective phase 2 trial. ${ }^{32}$ Many questions exist, and the best way to apply this exciting treatment approach is not yet clear. Most series use regimens from the RTOG 90-05 trial; but it is not clear if this is the ideal regimen when a tumor no longer exists. ${ }^{33}$ In addition, in cases in which an incomplete resection has been performed or a tumor has recurred in the resection cavity before SRS, the ideal treatment approach is unknown.

We present preliminary results of a new treatment approach using postoperative SRS for residual or recurrent brain metastases by applying a low dose to the resection bed and simultaneously integrating a boost dose to the gross tumor volume (GTV).

\section{Methods and materials}

Since 2010, our institution has offered postoperative radiosurgery as an alternative to WBRT to patients who have undergone surgery for a dominant metastatic focus and had limited synchronous metastatic disease. We reviewed all cases of postoperative SRS delivery and identified 3 cases in which different dose prescriptions were used. These cases were treated during 2011-2014.

Radiosurgery was delivered using the Trilogy Linear Accelerator (Varian Medical Systems, Palo Alto, CA) and the CyberKnife System (Accuray Inc, Sunnyvale, CA). Treatment was frameless, and thermoplastic masks were used for patient immobilization. Optical guidance with an infrared mouthpiece was used with the Trilogy for setup verification. Orthogonal $\mathrm{kV}$ X-ray imaging was used with the CyberKnife System. All of the patients underwent gadolinium-enhanced magnetic resonance (MR) imaging of the brain within 48 hours of surgical resection to delineate any residual disease and assess the quality of the resection. Another MR of the brain was done and a computed tomography $(\mathrm{CT})$ simulation was created using a $1.5-\mathrm{mm}$ slice interval. Both MR images were registered and fused with CT data from the simulation using either the Varian Eclipse Treatment Planning System or the Accurary CyberKnife system. Segmentation was performed by our institution's radiation oncologist, neurosurgeon, and neuroradiologist. For each partially resected metastasis, 2 target volumes were delineated. The clinical target volume (CTV) resection cavity is the MR-defined resection cavity with no added margin. The GTV is the MR-defined residual disease based on both the immediate postoperative and treatment planning MR images. No additional margin was added on the CTV or GTV for treatment planning. Prescription doses varied between the 3 treated patients, but ranged from 4-15 Gy for the CTV and 17-18 Gy for the GTV prescribed to the 71\%$78 \%$ isodose line. For patients who were treated with the CyberKnife, a sum plan was used to combine the dose from both targets. Using the Eclipse Treatment Planning System, it was possible to construct a single plan. Patients were seen in follow-up every 2-3 months with new MR brain imaging.

We constructed a traditional postoperative radiosurgery plan by prescribing the higher definitive dose to the larger CTV target. This new plan was compared with the simultaneous integrated boost (SIB) plan, which was originally delivered. Dosimetric information was collected from the treatment planning systems and included minimum, maximum, and mean doses for the cavity and residual tumor, the volume of brain receiving $12 \mathrm{~Gy}$ (V12Gy), and conformity indices. Values were compared using a paired $t$ test.

\section{Results}

\section{Clinical courses of patients treated with technique}

There were 3 patients treated at our institution using this technique. Patient characteristics and indications for surgical resection are listed in Table 1. The first patient treated with this technique presented with headaches, visual field deficits, and right-sided neglect. Imaging of the brain showed a 3.1-cm rim-enhancing lesion in the left parietal lobe with vasogenic edema and $1 \mathrm{~cm}$ of subfalcine herniation. Further workup revealed a likely primary mass in the right kidney. The patient underwent a left parietal craniotomy and resection of the mass, which was found on pathology to be consistent with renal cell carcinoma. A postoperative MRI showed expected postsurgical changes and no residual disease in the resection cavity; however, 1 month after surgical resection, the MR brain image used for SRS planning revealed a small local recurrence at the posterior aspect of the previously resected cavity. A SIB plan was developed with 15 Gy prescribed to the larger postresection cavity and $18 \mathrm{~Gy}$ to the gross tumor, both prescribed to the $74 \%$ isodose line (Figures 1 and 2). Treatment was delivered without complication, and follow-up imaging confirmed resolution of the contrast-enhancing region of the resection cavity. Subsequent imaging confirmed local response but showed distant brain failure with multiple new lesions. The patient underwent WBRT 4 months after the postoperative SRS with SIB.

The second patient presented to the emergency department with left facial droop. Imaging revealed a 3-cm 


\section{Original Report}

\begin{tabular}{|cccccc|}
\hline TABLE 1 Patient characteristics & Age $(\mathbf{y})$ & Sex & Primary site & RPA class & $\begin{array}{c}\text { Reason for } \\
\text { surgery }\end{array}$ \\
\hline 1 & 49 & $M$ & Kidney & 2 & $\begin{array}{c}\text { Emergent } \\
\text { decompression }\end{array}$ \\
2 & 76 & $M$ & Lung & 2 & $\begin{array}{c}\text { Emergent } \\
\text { decompression }\end{array}$ \\
3 & 50 & M & Lung & 2 & $\begin{array}{c}\text { Symptomatic with } \\
\text { steroids }\end{array}$ \\
\hline
\end{tabular}

RPA, recursive partitioning analysis

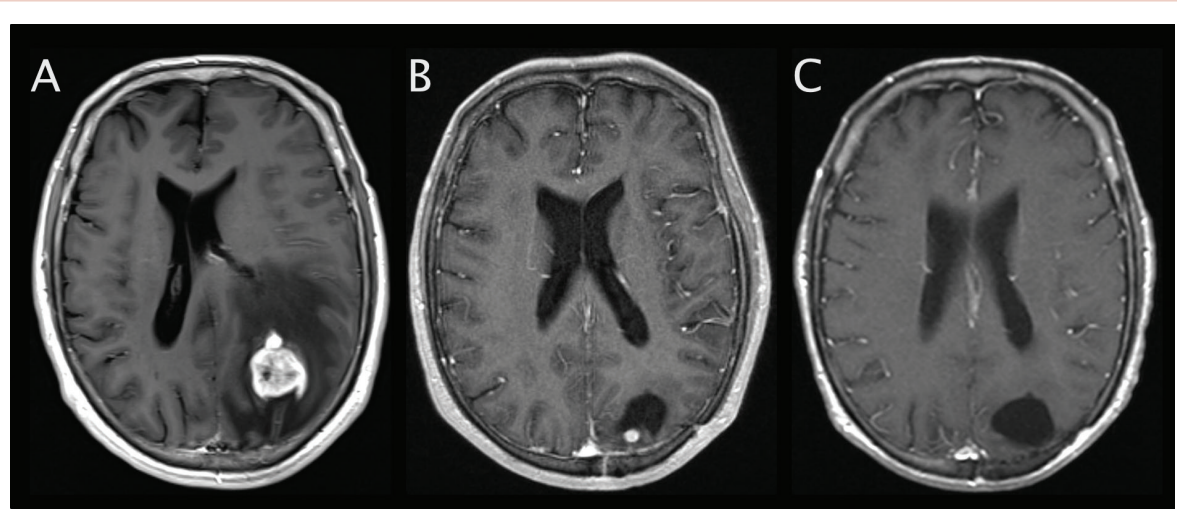

FIGURE 1 A, Magnetic-resonance brain scan demonstrating preoperative tumor. B, Stereotactic radiosurgery planning imaging after resection. C, Resolution of nodular contrast enhancement.

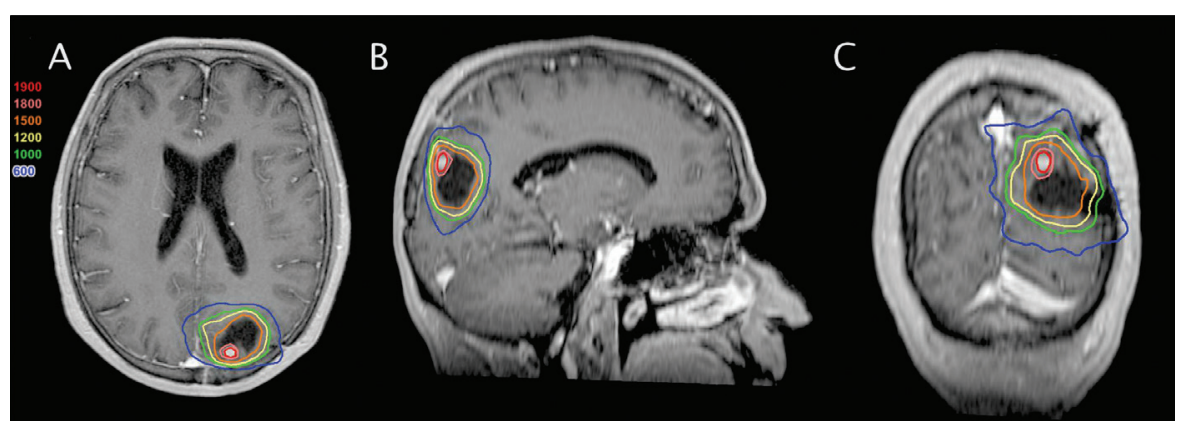

FIGURE 2 Stereotactic radiosurgery plan demonstrating simultaneous integrated boost technique in axial (A), sagittal (B), and coronal $(C)$ planes. tion of the contrast enhancement in the resection cavity. Six months after SRS, there was no evidence of disease.

The third patient presented to his primary care physician with a right-sided headache. MR imaging of the brain showed a $1.7-\mathrm{cm}$ enhancing lesion in the right frontal lobe. Steroids did not improve his symptoms, and the patient underwent a craniotomy and gross total resection of the metastasis based on the neurosurgeon's intraoperative observations and postoperative imaging. Follow-up imaging showed an area of nodular enhancement felt to be consistent with recurrent disease. The postoperative cavity was treated to 15 Gy with a SIB of $17 \mathrm{~Gy}$ to the area of residual disease using SRS prescribed to the $71 \%$ isodose line. The patient tolerated SRS well with only a slight headache for several days. Follow-up imaging revealed distant brain failure, which was treated 3 months after the original SRS. There was no evidence of intracranial disease 6 months after SRS.

\section{Clinical outcomes}

All treated lesions responded to SRS with SIB. No local recurrence has been observed in or adjacent to the postoperative cavity in any of the patients treated with this technique. However, 2 of the 3 patients developed distant brain failure after SRS with SIB (mean, 3.5 months). Of the 2 patients, 1 was salvaged with traditional SRS for a new lesion, and 1 patient was treated with palliative WBRT for multiple new lesions. None of the patients who were treated with this technique developed grade 3 or greater clinical toxicity, and no imaging findings consistent with radionecrosis have been observed. enhancing mass in the right temporal lobe with $4 \mathrm{~mm}$ of midline shift secondary to vasogenic edema. The patient was taken for a right frontotemporal craniotomy with image guidance and microdissection, but complete resection was impossible because of the tumor's proximity to the middle cerebral artery. The patient did well after surgery, and an SRS with SIB plan was constructed. The resection cavity received $15 \mathrm{~Gy}$, and the focal area of residual disease received $18 \mathrm{~Gy}$ prescribed to the $78 \%$ isodose line. The patient did well, and follow-up imaging confirmed resolu-

\section{Dosimetric outcomes}

Dosimetric comparisons of SRS with SIB plans and traditional postoperative SRS plans are presented in Table 2 and Figure 3. The V12Gy was significantly reduced using SIB plans compared with traditional postoperative SRS (mean, 15.6 vs $20.0 \mathrm{~cm}^{3} ; P=.04$ ). There were no differences in the maximum dose delivered to either the tumor $(P=.15)$ or cavity $(P=.13)$ using the 2 plans. The average mean tumor dose was 17.86 Gy using the SIB plan, compared with $20.38 \mathrm{~Gy}$ using the traditional plan $(P=$ 


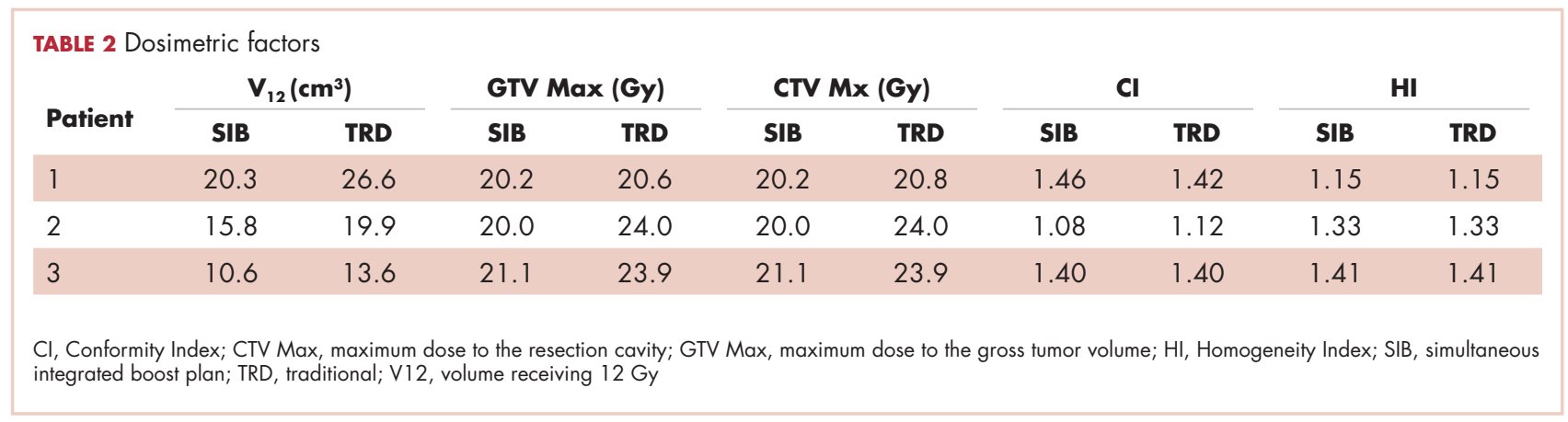

.03). The average mean cavity dose was $16.20 \mathrm{~Gy}$ using the SIB plan, compared with 19.71 Gy using the traditional plan $(P=.05)$. No statistically significant differences were observed in the Conformity Index, New Conformity Index, or Homogeneity Index. In both of the patients who were treated with the CyberKnife linear accelerator (where treatment times can be calculated), the treatment times were similar for delivery of the 2 plans (SRS with $\mathrm{SIB}$ and SRS alone; 43 vs 30 minutes and 53 vs 55 minutes, respectively).

\section{Discussion}

There has been increasing interest in using SRS after surgery in place of traditional WBRT as an adjuvant to surgery. SRS can offer similar postoperative local control with only a single treatment and reduce acute toxicity for many patients. However, perhaps the most exciting benefit of postoperative SRS is the potential reduction in neurocognitive side effects seen with WBRT. Investigators continue to better define dosing schedules, target delineation techniques, and timing of postoperative SRS. ${ }^{34}$

Residual or recurrent disease can present a management challenge in postoperative SRS. Brennan and colleagues observed that 3 patients out of 49 had local recurrence in the surgical resection cavity in the interim between surgery and SRS. ${ }^{32}$ Rates of subtotal resections in a postoperative SRS series vary between $75 \%$ and $100 \% \cdot{ }^{13-22,24,25,28,30,35}$ Taken together, the risk of a patient having either residual disease following surgery or recurrent disease that developed in the treatment interval is a significant problem. In our series, 2 patients were treated for recurrent disease, and 1 patient was treated for a subtotal resection. Our treatment approach did not differ for these 2 groups of patients. Both groups likely resulted from residual disease (either clinically recognized or not) with continued progression.

Without the SIB technique, in cases of persistent or recurrent disease, a high dose is traditionally prescribed to the entire cavity either with or without an added margin. ${ }^{13-22,24,25,28,30}$ This approach results in a compromise of dose, typically less than would be used for an intact

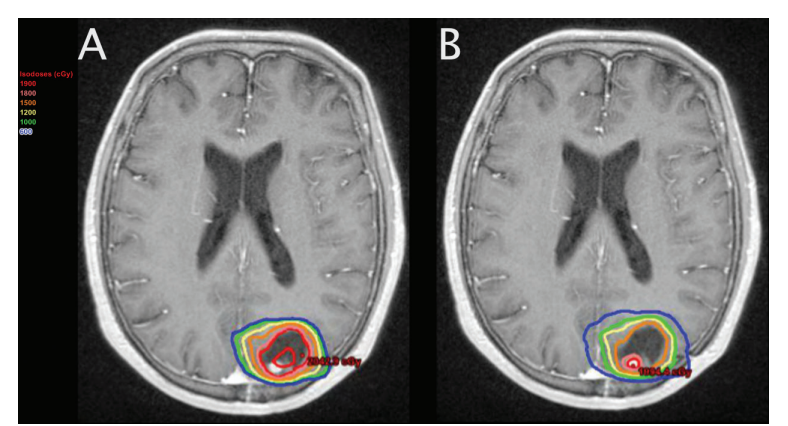

FIGURE 3 Comparison of traditional stereotactic radiosurgery plan (A) and simultaneous integrated boost plan (B).

metastasis of a similar size and greater than what is needed for control of the postresection cavity. Hartford and colleagues have demonstrated adequate local control of $84 \%$ with a median marginal dose of only $10 \mathrm{~Gy}^{15}$ Although authors state that their typical dose is now higher; it is clear that in postoperative SRS, an inadequate dose is not the primary factor that drives recurrence. Rogers and colleagues reported on the use of the GliaSite system (IsoRay Medical, Richland, Washington, USA) for treatment of the resection cavity after surgical resection of brain metastases. ${ }^{36}$ Despite doses of $60 \mathrm{~Gy}$ at $1 \mathrm{~cm}$, authors observed local control of 13\%-18\%. It seems clear that dose escalation is not the answer to improving local recurrence after adjuvant treatment of resected brain metastases.

In the absence of data demonstrating the necessity of higher doses, all efforts should be made to reduce toxicity in patients treated for brain metastases. The rate of observed radionecrosis was $17.5 \%$ in a study by Brennan and colleagues. ${ }^{32}$ It can be difficult to predict which patients will develop radionecrosis after SRS, but it seems to be related to dose and volume. ${ }^{33}$ The volume of the brain receiving 12 Gy (V12Gy ) in a single fraction has been shown to predict the development of radionecrosis. ${ }^{37,38}$ This V12Gy rapidly increases with even slightly larger target volumes. By using the SIB technique, an adequate dose can be given to the residual disease with a minimum increase in volume of the normal brain at risk for radionecrosis. 


\section{Conclusion}

In patients with recurrent or residual disease following surgical resection, SRS using a SIB is technically feasible and safe. This technique places a smaller volume of normal brain tissue at risk for radionecrosis, which may result in less patient toxicity. More patients and longer follow-up are required to better assess the clinical outcomes following treatment with this new technique.

\section{References}

1. Nayak L, Lee EQ, Wen PY. Epidemiology of brain metastases. Curr Oncol Rep. 2012;14:48-54.

2. Takakura K, Sano K, Hojo S, Hirano A, eds. Metastatic tumors of the central nervous system. Tokyo, Japan; New York, NY: IgakuShoin; 1982.

3. Fabi A, Felici A, Metro G, et al. Brain metastases from solid tumors: disease outcome according to type of treatment and therapeutic resources of the treating center. J Exp Clin Cancer Res: CR. 2011;30:10.

4. Nussbaum ES, Djalilian HR, Cho KH, Hall WA. Brain metastases. Histology, multiplicity, surgery, and survival. Cancer. 1996;78:1781-1788.

5. Andrews DW, Scott CB, Sperduto PW, et al. Whole brain radiation therapy with or without stereotactic radiosurgery boost for patients with one to three brain metastases: phase III results of the RTOG 9508 randomised trial. Lancet. 2004;363:1665-1672.

6. Noordijk EM, Vecht CJ, Haaxma-Reiche H, et al. The choice of treatment of single brain metastasis should be based on extracranial tumor activity and age. Int J Radiat Oncol Biol phys. 1994;29:711-717.

7. Patchell RA, Tibbs PA, Walsh JW, et al. A randomized trial of surgery in the treatment of single metastases to the brain. N Eng J Med. 1990;322:494-500.

8. Aoyama H, Shirato H, Tago M, et al. Stereotactic radiosurgery plus whole-brain radiation therapy vs stereotactic radiosurgery alone for treatment of brain metastases: a randomized controlled trial. JAMA 2006;295:2483-2491.

9. Chang EL, Wefel JS, Hess KR, et al. Neurocognition in patients with brain metastases treated with radiosurgery or radiosurgery plus whole-brain irradiation: a randomised controlled trial. Lancet Oncol. 2009;10:1037-1044.

10. Kocher M, Soffietti R, Abacioglu U, et al. Adjuvant whole-brain radiotherapy versus observation after radiosurgery or surgical resection of one to three cerebral metastases: results of the EORTC 2295226001 study. J Clin Oncol. 2011;29:134-141.

11. Patchell RA, Tibbs PA, Regine WF, et al. Postoperative radiotherapy in the treatment of single metastases to the brain: a randomized trial. JAMA. 1998;280:1485-1489.

12. Choi CY, Chang SD, Gibbs IC, et al. What is the optimal treatment of large brain metastases? An argument for a multidisciplinary approach. Int J Radiat Oncol Biol Phys. 2012;84:688-693.

13. Choi CY, Chang SD, Gibbs IC, et al. Stereotactic radiosurgery of the postoperative resection cavity for brain metastases: prospective evaluation of target margin on tumor control. Int J Radiat Oncol Biol Phys. 2012;84:336-342.

14. Do L, Pezner R, Radany E, Liu A, Staud C, Badie B. Resection followed by stereotactic radiosurgery to resection cavity for intracranial metastases. Int J Radiat Oncol Biol Phys. 2009;73:486-491.

15. Hartford AC, Paravati AJ, Spire WJ, et al. Postoperative stereotactic radiosurgery without whole-brain radiation therapy for brain metastases: potential role of preoperative tumor size. Int J Radiat Oncol Biol Phys. 2013;85:650-655.

16. Hwang SW, Abozed MM, Hale A, et al. Adjuvant Gamma Knife radiosurgery following surgical resection of brain metastases: a 9-year retrospective cohort study. J Neurooncol. 2010;98:77-82.

17. Iwai Y, Yamanaka K, Yasui T. Boost radiosurgery for treatment of brain metastases after surgical resections. Surg Neurol. 2008;69:181186; discussion 186.

18. Jagannathan J, Yen CP, Ray DK, et al. Gamma Knife radiosurgery to the surgical cavity following resection of brain metastases. J Neuro- surg. 2009;111:431-438.

19. Jensen CA, Chan MD, McCoy TP, et al. Cavity-directed radiosurgery as adjuvant therapy after resection of a brain metastasis. J Neurosurg. 2011;114:1585-1591.

20. Karlovits BJ, Quigley MR, Karlovits SM, et al. Stereotactic radiosurgery boost to the resection bed for oligometastatic brain disease: challenging the tradition of adjuvant whole-brain radiotherapy. Neurosurg Focus. 2009;27:E7.

21. Kelly PJ, Lin YB, Yu AY, et al. Stereotactic irradiation of the postoperative resection cavity for brain metastasis: a frameless linear accelerator-based case series and review of the technique. Int J Radiat Oncol Biol Phys. 2012;82:95-101.

22. Limbrick DD Jr, Lusis EA, Chicoine MR, et al. Combined surgical resection and stereotactic radiosurgery for treatment of cerebral metastases. Surg Neurol. 2009;71:280-288, discussion 288-289.

23. Luther N, Kondziolka D, Kano H, et al. Predicting tumor control after resection bed radiosurgery of brain metastases. Neurosurgery. 2013;73:1001-1006; discussion 1006.

24. Mathieu D, Kondziolka D, Flickinger JC, et al. Tumor bed radiosurgery after resection of cerebral metastases. Neurosurgery. 2008;62:817-823; discussion 823-814.

25. Minniti G, Esposito V, Clarke E, et al. Multidose stereotactic radiosurgery $(9 \mathrm{~Gy}$ x 3$)$ of the postoperative resection cavity for treatment of large brain metastases. Int J Radiat Oncol Biol Phys. 2013;86:623-629.

26. Ogiwara H, Kalakota K, Rakhra SS, et al. Intracranial relapse rates and patterns, and survival trends following post-resection cavity radiosurgery for patients with single intracranial metastases. J Neurooncol. 2012;108:141-146.

27. Prabhu R, Shu HK, Hadjipanayis C, et al. Current dosing paradigm for stereotactic radiosurgery alone after surgical resection of brain metastases needs to be optimized for improved local control. Int J Radiat Oncol Biol Phys. 2012;83:e61-66.

28. Quigley MR, Fuhrer R, Karlovits S, Johnson M. Single session stereotactic radiosurgery boost to the post-operative site in lieu of whole brain radiation in metastatic brain disease. J Neurooncol. 2008;87:327-332.

29. Robbins JR, Ryu S, Kalkanis S, et al. Radiosurgery to the surgical cavity as adjuvant therapy for resected brain metastasis. Neurosurgery. 2012;71:937-943.

30. Soltys SG, Adler JR, Lipani JD, et al. Stereotactic radiosurgery of the postoperative resection cavity for brain metastases. Int J Radiat Oncol Biol Phys. 2008;70:187-193.

31. Wang CC, Floyd SR, Chang CH, et al. Cyberknife hypofractionated stereotactic radiosurgery (HSRS) of resection cavity after excision of large cerebral metastasis: efficacy and safety of an $800 \mathrm{cGy} \times 3$ daily fractions regimen. J Neurooncol. 2012;106:601-610.

32. Brennan C, Yang TJ, Hilden P, et al. A phase 2 trial of stereotactic radiosurgery boost after surgical resection for brain metastases. Int J Radiat Oncol Biol Phys. 2014;88:130-136.

33. Shaw E, Scott C, Souhami L, et al. Single dose radiosurgical treatment of recurrent previously irradiated primary brain tumors and brain metastases: final report of RTOG protocol 90-05. Int J Radiat Oncol Biol Phys. 2000;47:291-298.

34. Amsbaugh MJ, Boling W, Woo SY. Tumor bed directed stereotactic radiosurgery for surgically resected brain metastases. Austin J Med Oncol. 2014;1:1-6.

35. Steinmann D, Maertens B, Janssen S, et al. Hypofractionated stereotactic radiotherapy (hfSRT) after tumour resection of a single brain metastasis: report of a single-centre individualized treatment approach. J Cancer Res Clin Oncol. 2012;138:1523-1529.

36. Rogers LR, Rock JP, Sills AK, et al; Brain Metastasis Study Group. Results of a phase II trial of the GliaSite radiation therapy system for the treatment of newly diagnosed, resected single brain metastases. J Neurosurg. 2006;105:375-384.

37. Korytko T, Radivoyevitch T, Colussi V, et al. 12 Gy gamma knife radiosurgical volume is a predictor for radiation necrosis in non-AVM intracranial tumors. Int J Radiat Oncol Biol Phys. 2006;64:419-424.

38. Minniti G, Clarke E, Lanzetta G, et al. Stereotactic radiosurgery for brain metastases: analysis of outcome and risk of brain radionecrosis. Radiat Oncol. 2011;6:48. 